\title{
Características socioganaderas y niveles de productividad de establos lecheros de la Irrigación Santa Rita en Arequipa
}

\author{
Characterize social-livestock situation and levels of productivity of dairy farms of Santa Rita \\ Irrigation at Arequipa
}

\author{
Agustín Pallete P. ${ }^{*}$, Annelisse Malaga N. ${ }^{2}$, María García S. ${ }^{3}$
}

* Autor de correspondencia

\begin{abstract}
Resumen
Esta investigación se realizó con la finalidad de evaluar la situación socioganadera y niveles de productividad de los establos participantes en el Servicio de Productividad Lechera de Arequipa. Las características socioganaderas se basan en encuestas realizadas, a 25 ganaderos, encontrándose que el 95\% son propietarios de sus parcelas que tuvieron una extensión promedio de 30,8 ha y que el $85 \%$ del ganado lechero que crían corresponde a la raza Holstein. El sistema de explotación en Santa Rita es 44\% tipo intensivo y el 56\% es mixto. El sistema de alimentación al pastoreo es el predominante con un 56\% y el 100\% de ganaderos suplementan con alimento balanceado. El $70 \%$ de ganaderos utilizan heno de alfalfa y el $40 \%$ proporcionan ensilado. La mayoría, 95\% utilizan sistema de ordeño mecánico. El 80\% mencionan el precio de la leche como su principal problema general. Los resultados de los aspectos productivos se basan en las evaluaciones de 9, 358 vacas, de las cuales 7,610 estuvieron en producción es decir el $81 \%$ del total, pertenecientes a 29 establos evaluados durante los años 2005 al 2010. Para el período de los seis años, las producciones fueron: General (Hato), 17,7 kg/día y por vaca 22,9 kg/día. Para los 29 establos las producciones fueron: General, 18,5 kg/día y por vaca, $22,8 \mathrm{~kg} /$ día. Las producciones de leche a través de los años estudiados muestran una tendencia positiva. Los índices productivos de Santa Rita se encuentran entre los más altos de Arequipa y son comparables favorablemente con otras cuencas lecheras del país.
\end{abstract}

Palabras clave: Leche; Social Ganaderas; Productividad.

\begin{abstract}
This research it has been made with the aim of evaluating the social livestock situation and the productivity levels of the dairy farms participants in the milk recording program of Arequipa. The social-livestock situation was evaluated based on a survey to 25 dairy farmers. It was found that $95 \%$ of farmers have their own parcels with an average area of 30,8 ha and that $85 \%$ of the cattle were Holstein breed. The $44 \%$ of the dairy farmer raise their cattle in an intensive system and $56 \%$ is a semi-intensive system. The food based on grazing it is the most common with $56 \%$, the alfalfa is used by $70 \%$ and silage by $40 \%$ of dairy farmers and all the farms use feed concentrate. The $95 \%$ of farmers have a milking machine, and $80 \%$ indicate that main problem is the price of milk. The results of the productivity parameters correspond to a total 9,358 dairy cows which 7,610 were on milking it is $81 \%$ of total owned to 29 dairy farmers in a six years from 2005 to 2010. For the six year period, the milk productions were: general $17,7 \mathrm{~kg}$ /day and per cow, $22.9 \mathrm{~kg} /$ day. For the 29 dairy farms the milk productions were: general, $18,5 \mathrm{~kg} /$ day and per cow 22,8 kg/day. The milk production per years shows us a positive trend. The productivity parameters of State Santa Rita stay among the highest levels of Arequipa and compare favourably with other dairy cows areas in the country.
\end{abstract}

Keywords: Milk; Social livestock; Productivity.

\section{Introducción}

La ganadería lechera en la Irrigación de Santa Rita de Siguas se inició en el año 1944, con una visión de ganadería moderna y empresarial, con ganado proveniente principalmente de la campiña de Arequipa, para posteriormente hacer importación de ganado Argentino y Uruguayo, hoy fuertemente influenciado por genética americana debido a la inseminación artificial. Su desarrollo se dio en propiedades que tenían 30 ha en promedio, en donde los ganaderos se establecieron junto con sus familias.

En la explotación lechera actual se requiere ser cada vez más eficiente, tanto productiva como reproductivamente, detectando problemas y planteando metas, es en este sentido que el uso de registros de productividad se ha convertido en una de las principales herramientas, necesarias en el manejo y evaluación de la información, en

\footnotetext{
1*Departamento de Producción Animal, Universidad Nacional Agraria La Molina, Lima, Perú. Email: aep@lamolina.edu.pe ${ }^{2}$ Universidad Católica de Santa María, Arequipa, Perú.

${ }^{3}$ Departamento de Producción Animal, Universidad Nacional Agraria La Molina, Lima, Perú. Email: megarcia@lamolina.edu.pe
} 
tal sentido la importancia de caracterizar una determinada región permite determinar sus parámetros de modo de poder identificarla y a la vez compararla. El objetivo del presente trabajo fue evaluar la situación socio-ganadera y niveles de productividad de la ganadería lechera de la Irrigación Santa Rita de Siguas en Arequipa.

\section{Materiales y métodos}

\section{Localización y duración}

Este estudio se realizó en la Irrigación de Santa Rita de Siguas, ubicada al noroeste de la provincia, departamento y región de Arequipa, a la altura del Km 931 de la carretera Panamericana Sur, entre las coordenadas $16^{\circ} 29^{\prime}$ '27" de latitud sur y $72^{\circ} 05^{\prime} 33^{\prime \prime}$ de longitud oeste. La altitud promedio alcanza los 1,268 m.s.n.m., posee un clima árido, seco, de alta temperatura, radiación y luminosidad solar. La humedad relativa en Santa Rita de Siguas durante el periodo de investigación estuvo en un rango de 18\% a $25 \%$, presentándose los mayores niveles entre enero y abril y los menores entre julio y setiembre. La temperatura media anual es de $20,5^{\circ} \mathrm{C}$, con una máxima de $32{ }^{\circ} \mathrm{C}$ en febrero y una mínima de $16,7^{\circ} \mathrm{C}$ en julio.

Para el presente trabajo se realizó la recolección de datos correspondientes a los años del 2005 al 2010; considerándose un total de 9,358 lactaciones, en 29 establos de la Irrigación Santa Rita participantes en el Servicio Oficial de Productividad Lechera (SOPL) de Arequipa.

\section{Fuentes de información}

- Encuestas realizadas durante el año 2010, para características socio-ganaderas de 25 ganaderos propietarios de establos que hacían uso del Servicio de Productividad Lechera en Santa Rita de Siguas.

- Base de Datos del Servicio Oficial de Productividad Lechera (SOPL) de Arequipa de 29 establos correspondientes a los años 2005, 2006, 2007, 2008, 2009 y 2010.

\section{Modelo Estadístico Lineal:}

$$
Y i j=u+T i+B j+e
$$

Dónde:

Yij = Valor de la observación correspondiente al i - esimo tratamiento (Establo) del $\mathrm{j}$ - esimo bloque (año).

$\mathrm{u} \quad=$ Media general.

$\mathrm{Ti}=$ Efecto del $\mathrm{i}$ - esimo tratamiento (establo)

$\mathrm{Bj}=$ Efecto del $\mathrm{j}$ - esimo bloque (año)

e $=$ Efecto del error

Los procedimientos estadísticos de los datos fueron realizados utilizando el paquete Statistical Analysis System (SAS).

\section{Resultados y discusión}

\section{Aspectos Socioganaderos}

Los resultados de las características socio-ganaderas se basan en las encuestas realizadas a 25 ganaderos propietarios de establos participantes en el Servicio Oficial de Productividad Lechera de Arequipa en la Irrigación de Santa Rita de Siguas, realizadas durante el año 2010.

\section{Propiedad de la tierra}

En la Irrigación de Santa Rita de Siguas se encontró que el $95 \%$ de los establos están establecidos en tierra de propiedad del mismo dueño, la mayoría de ellos la heredaron junto con la crianza de ganado lechero y el 5\% están establecidos en parcelas alquiladas. Estos porcentajes se asemejan mucho a los encontrados por Medina (1988) de 94\%, Valenza (1991) de 95\%, ambos para la cuenca de Arequipa y por Bernal (1993) para las Irrigaciones de Majes y Santa Rita de Siguas de $85 \%$ de propietarios de la tierra en la que desarrollaban ganadería lechera.

La confianza que da el saberse dueño de la tierra es fundamental para poder desarrollarse con tranquilidad y así el ganadero siente mayor seguridad al intervenir, crecer y ser cada vez más eficiente económica y técnicamente, sobre todo teniendo en cuenta que en lechería se debe pensar a largo plazo, además que la posesión del terreno le brinda acceso a créditos algunas veces indispensables para la inversión.

\section{Extensión de las parcelas}

El tamaño de las propiedades donde se encuentran los establos de los ganaderos encuestados varió entre 5 y 90 ha, siendo el promedio 30,8 ha y conservan una cantidad considerable de terreno destinada a cultivos forrajeros, $32 \%$ de estos tienen extensiones mayores a 40 hectáreas, considerándose estos fundos mixtos, ya que no solo se dedican a la ganadería sino también a la producción agroalimentaria, siendo los cultivos que ocupan la mayor extensión los de alcachofa, esparrago, cebolla, ají paprika, pimiento, frijol y frutales como el melón, la palta y la uva, cuyos restos de cosecha forman parte de la ración, principalmente de la recría.

Las propiedades de Santa Rita de Siguas, a diferencia de las parcelas de la Irrigación de Majes que son de cinco hectáreas, tienen la ventaja del tamaño, el cual permite a los establos tener mayor cantidad de animales pudiendo autoabastecerse de forraje.

El 80\% de los propietarios vive en Santa Rita de Siguas, el $40 \%$ de ellos lo hace con su familia. Este resultado se asemeja mucho al encontrado por Pimentel (1994) que afirma que el $80 \%$ de los colonos de la sección "A" de la Irrigación de Majes, viven con su familia en la parcela. Siendo porcentajes altos en ambos casos, lo que demuestra el arraigo que tiene el ganadero con respecto a la lechería.

\section{Fuerza laboral}


La mano de obra en los establos de Santa Rita depende del número de animales y de la tecnificación con la que cuenten. Se encontró que la mano de obra familiar no rentada representa el $40 \%$, este valor es menor al encontrado por Valenza (1991) que encontró que en Arequipa el $51,9 \%$ de la mano de obra era familiar no rentada. Los ganaderos encuestados que afirman que la participación de sus familias en la ganadería lechera disminuyó fueron los que poseen los establos con menor número de animales, ya que sus hijos prefieren dedicarse a otros rubros como el comercio porque consideran a la ganadería como un trabajo muy sacrificado. Sin embargo en los establos con mayor número de animales los ganaderos afirman que ha aumentado la participación familiar, encontrando que los hijos de estos, en gran proporción se inclinan por profesiones y carreras técnicas afines a la ganadería, siendo su participación muchas veces indirecta.

\section{Sistemas de crianza}

El predominio en el ganado vacuno lechero en Santa Rita es de la raza Holstein Friesian, representando el $85 \%$ del total del ganado, viene siendo criado en la zona desde hace más de 40 años, procediendo dela campiña de Arequipa, el que luego se vio influenciado por animales de origen holandés, argentino, americano, uruguayo y canadiense, ya sea por importación de animales o de semen. El predominio de la raza Holstein es el mismo que el reportado por Medina (1988) en Arequipa, pero el porcentaje de este es ampliamente mayor al encontrado de $65 \%$ de predominancia de Holstein, al igual que el reportado por Valenza (1991) de predominancia Holstein con 54\%; pero menor al reportado por Bernal (1993) con $93 \%$ en Arequipa. Entre el $15 \%$ de las otras razas que se encontraron esta la introducción de razas lecheras como la raza Jersey y la Brown Swiss entre otras, generalmente cruzadas con la raza Holstein Friesian para aprovechar características como la de facilidad al parto, vigor híbrido y porcentaje de grasa en leche.

El 97\% de los ganaderos hace recría de sus animales, quedándose con las crías hembras para reemplazos, y el $3 \%$ reemplaza mediante la compra de vaquillas y en muy pocas ocasiones con vacas de primer parto. El 50\% de los ganaderos encuestados afirma que selecciona la recría basándose en la producción lechera de la madre y tomando en cuenta los valores estimados de habilidad transmisora para producción de leche (PTA) del padre, el 45\% selecciona la recría considerando además características de exterior, siendo la característica más determinante para ellos el peso al nacimiento y el 5\% selecciona únicamente por características fenotípicas del animal.

Mientras que el 55\% hace saca de los terneros machos al nacer, luego de ingerir calostro (dos días), en el $45 \%$ de los establos se cría a los terneros machos hasta el destete y en algunos casos hasta los dos años; esto se da mayormente en los establos con menor número de animales. Los terneros son destinados a la venta, el 5\% de ellos son seleccionados para toros repasadores para los establos de la irrigación en los que se emplea la monta natural, en un sistema mixto con inseminación artificial y un gran porcentaje de estos son llevados a establos fuera de la irrigación.

La saca promedio de hembras es de $2 \%$, siendo la causa principal problemas reproductivos, lesiones de ubre por mastitis, y otras menos importantes. Los ganaderos de algunos establos (3\%) manifestaron interés por erradicar enfermedades como la neospora y leptospira, las cuales ocasionan perdidas económicas, por lo que hacen análisis frecuentes y la posterior saca gradual de las vacas positivas

En el 44\% de los establos el sistema de explotación es del tipo intensivo y en el $56 \%$ de establos es semi intensivo o mixto. La elección del tipo de explotación depende fundamentalmente del número de animales, en establos pequeños es más común ver el uso del sistema mixto, donde los animales salen de los corrales para ir a pastorear y vuelven para el suministro de alimento balanceado y el ordeño. Los resultados encontrados en Santa Rita de Siguas difieren de los reportados por Medina (1988) y por Valenza (1991) en Arequipa, encontrando que el sistema de explotación intensivo tuvo promedios de $3 \%$ y de $2 \%$ respectivamente, esta diferencia se puede deber a que en la Irrigación de Santa Rita de Siguas el promedio de vacas por establo es mayor que en otras zonas de Arequipa y han optado por el sistema intensivo el cual les da mayores ventajas.

En el 70\% de los establos los animales son separados en corrales por clase, es decir, vacas en producción, vacas en seca, vaquillonas, terneras, etc.; en el 30\% de ellos además separan a las vacas por nivel de producción, es decir, vacas de alta, mediana y baja producción. En establos con menor número de animales en algunos casos se utiliza el mismo corral para vacas en seca y vaquillonas y no se hace distinción entre vacas por su nivel de producción.

\section{Alimentación}

En los establos evaluados se encontró que el 56\% alimentan al ganado usando el sistema de pastoreo sea este total o parcial (cuando parte del forraje se ofrece en comedero) y el $44 \%$ tienen a las vacas estabuladas permanentemente y les ofrecen el alimento en el comedero. El 73\% les suministra el forraje y una suplementación de alimento balanceado por separado, mientras que el $27 \%$ les ofrece el alimento en la forma de TMR (ración total mezclada) en la que se mezcla de forma uniforme y completa el forraje y el concentrado. Se observó que en los establos pertenecientes a propiedades mixtas con agroindustria, los restos de cosecha fueron evaluados y procesados para formar parte de la ración, estos fueron utilizados sobre todo para la alimentación de la recría y vacas en seca, ya que algunos de sus componentes cambian las características organolépticas de leche como es el olor y en algunos casos el sabor.

El $100 \%$ de los encuestados aseguraron que suministraban alimento balanceado en sus establos, la cantidad y los insumos utilizados para la preparación de las raciones variaron entre establos; el 25\% suministró 
alimento balanceado durante el ordeño. El $98 \%$ de ellos se lo brindo a terneras de cuna, el $55 \%$ proporcionó alimento balanceado a la recría y el $35 \%$ variaba la cantidad y los insumos para la preparación de este dependiendo del nivel de producción. De los establos encuestados el $85 \%$ prepara los alimentos balanceados en el establo o lo manda a preparar en lugares especializados y el otro $15 \%$ compra alimento balanceado comercial.

El 70\% de los ganaderos encuestados afirmó utilizar heno de alfalfa como parte de la ración, siendo el $20 \%$ de este preparado en el establo y el $80 \%$ lo compran, utilizándolo mayormente en los establos con ganado estabulado.

El $40 \%$ mostraron que sus establos contaban con silos de material noble, el 57\% tenían una zona destinada para ensilar mediante la colocación de material plástico directamente sobre el suelo y el 3\% que compró el silaje. El principal problema que encontraron en el uso de silos fue la exposición a la oxidación y la proliferación de hongos ya que el ensilado no es retirado de la forma correcta por lo que muchas veces pierde el valor nutritivo y en otros tiene que ser botado lo que ocasiona pérdidas económicas.

En cuanto a la disponibilidad de agua, se encontró que en animales bajo el sistema de explotación intensiva (44\%), un $80 \%$ contaban con bebederos que suministran agua fresca, ad libitum y $20 \%$ tuvieron algunas carencias en cuanto a la cantidad de esta.

En los animales que se encuentran bajo el sistema de explotación mixta (56\%) se encontró mayores problemas en cuanto al suministro de agua, ya el $95 \%$ de estos tenían bebedero dentro del corral, sin embargo ofrecían agua a los animales solo en horas de la tarde cuando regresaban para el ordeño; teniendo en cuenta la temperatura del ambiente, que durante el día puede llegar hasta los $32{ }^{\circ} \mathrm{C}$ en verano y que las vacas en producción requieren consumir gran cantidad de agua para la producción de leche, la falta de agua ocasiona estrés y repercute directamente sobre la producción; el 5\% que no tienen bebedero en el corral solo ofrecen agua al momento del ordeño, este hecho trae consecuencias graves en cuanto a desarrollo y producción de leche.

\section{Sistemas de ordeño}

Las salas de ordeño que se observaron en la zona, van desde salas básicas provistas generalmente de comederos, hasta salas tecnificadas conectadas a la zona de enfriamiento de leche.

Se encontró que en el 5\% de los establos se hacía el ordeño de forma manual, los ganaderos que así lo hacen afirman que para el número de vacas en ordeño, al ser menor de 15 , no justifica la adquisición de una máquina ordeñadora. Esta situación es similar a la encontrada por Casapía (2001) en la Irrigación de Majes, en la que los ganaderos de minifundios, con menos de 15 vacas, afirmaron que el número de vacas en producción no justificaba la inversión en salas de ordeño tecnificadas.

El otro $95 \%$ utilizan máquina ordeñadora, el tamaño y la tecnología de estas depende sobre todo del número de animales a ordeñar; en el $25 \%$ de los establos se hace tres ordeños, en el 10\% de estos a todas las vacas en producción y en el $15 \%$ solo para vacas de alta producción.

\section{Registros pecuarios}

El presente trabajo de investigación se realizó con los establos participantes en el Servicio de Productividad Lechera de Arequipa. Este brinda a los ganaderos registros productivos mensuales, los que son de gran importancia ya que sirven para hacer evaluaciones en forma individual y colectiva, además que en muchos establos esta es la única información existente; en el 50\% de los establos encuestados se encontró que llevaban además controles diarios de producción.

La falta de información, sobre todo de los índices reproductivos, en los establos que no llevan registro de acontecimientos y de su evaluación dificulta hacer un análisis correcto de estos establos, lo que no les permite plantear metas concretas y detectar problemas lo que conlleva una situación técnica-económica muy problemática.

\section{Problemática general}

El $80 \%$ de los entrevistados siente que el problema principal de los ganaderos es el precio de la leche, este resultado es similar al encontrado por Pimentel (1994) de $77 \%$ en la Irrigación de Majes en la sección “A”. Para poder sobrellevar este problema es necesario que el ganadero se vuelva cada vez más eficiente, reduciendo los costos de producción y aplicando tecnología con el fin de obtener un mayor margen de ganancia.

El 17\% señaló tener problemas para acceder a mano de obra indirecta, médico veterinario y técnico inseminador, además de no poseer tanque de nitrógeno para almacenaje de pajillas por lo que dependían del stock limitado que se les presentaba en el momento de la inseminación, la mayoría de veces sin especificaciones técnicas del semen y esto redujo las posibilidades de hacer un buen uso técnicogenético.

Los costos de producción representaron el 3\% de la problemática de los ganaderos de Santa Rita, este porcentaje es menor que el encontrado por Pimentel (1994) en la Irrigación de Majes en la sección "A", de 16\%; esta diferencia podría deberse a que en Majes, por el tamaño de las parcelas tienen limitada producción de forrajes, por lo que debieron comprarlo para la alimentación del ganado, situación que no ocurre en Santa Rita donde el costo que tuvieron en forrajes fue bajo debido a que el tamaño de las propiedades les permite autoabastecerse.

\section{Aspectos Productivos}

Para el análisis de los índices productivos se evaluaron las producciones de leche de 9,358 vacas provenientes de 29 establos participantes en el Servicio Oficial de Productividad Lechera de Arequipa en la Irrigación de 
Santa Rita de Siguas, en un período de seis (6) años, del 2005 al 2010. Los resultados se presentan según se indica a continuación en la Tabla 1 y 2

\section{Cantidad total de vacas \\ Por años}

En el Tabla 1 se muestran las cantidades totales de vacas del periodo de años del 2005 al 2010, estas fueron 1,299, $2,030,1,420,1,588,1,487$ y 1,534 vacas respectivamente, siendo el menor número de vacas de 1,299 en el año 2005 y el número máximo fue de 2,030 vacas en el año 2006, la diferencia en este índice se puede deber a que en el año 2006 se realizó una importación de hembras provenientes de Argentina y Uruguay, que fueron utilizados como reemplazo, generando una mayor cantidad de vacas en el año 2006. Luego por procesos de selección y descarte de vacas estos disminuyeron pero manteniendo un nivel superior al año 2005. El total de vacas incluidas en este estudio fue de 9,358 para el período de los seis (6) años. Los resultados del número total de vacas por años, en el periodo 2005 al 2010 tuvieron diferencia altamente significativa $(\alpha=0.01)$.

Tabla 1. Índice productivo por años

\begin{tabular}{ccccccc}
\hline \multirow{2}{*}{ Año Establos } & \multicolumn{3}{c}{ Cantidad de Vacas } & \multicolumn{2}{c}{$\begin{array}{c}\text { Producción de } \\
\text { Leche }\end{array}$} \\
\cline { 3 - 7 } & \multicolumn{3}{c}{ Total Producción Porcentaje } & $\begin{array}{c}\text { General Por vaca } \\
\text { Kg }\end{array}$ & Kg \\
\hline 2005 & 23 & 1,299 & 1,117 & 86 & 14.1 & 22.3 \\
2006 & 24 & 2,030 & 1,322 & 65 & 14.5 & 22.2 \\
2007 & 26 & 1,420 & 1,249 & 88 & 19.3 & 21.5 \\
2008 & 29 & 1,588 & 1,345 & 85 & 19.3 & 22.5 \\
2009 & 28 & 1,487 & 1,256 & 84 & 20.0 & 23.7 \\
2010 & 27 & 1,534 & 1,321 & 86 & 19.6 & 25.1 \\
\hline
\end{tabular}

En la Figura 1 se puede apreciar las tendencias, para la cantidad de vacas total y en producción, por año para el total del periodo de seis años y que en general son ascendentes, es decir la población de vacas evaluadas fue en aumento.

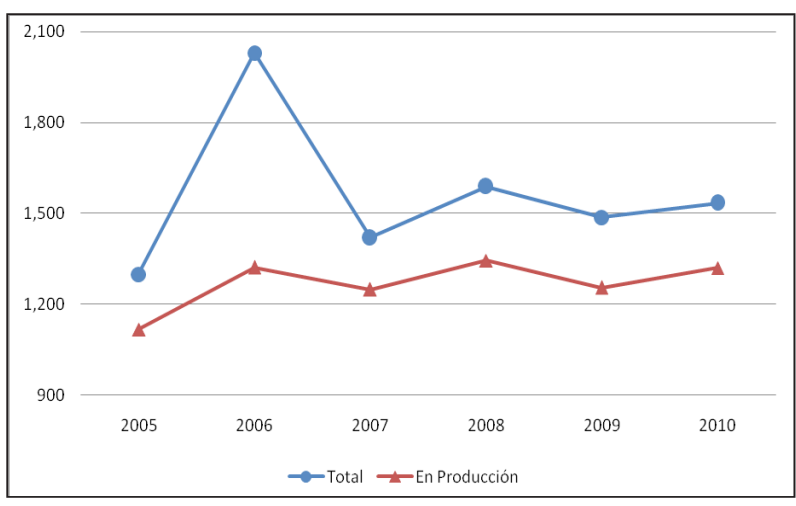

Figura 1. Cantidad de vacas por año
En el Tabla 2 se puede apreciar la cantidad total de vacas por establo, encontrándose que el promedio de vacas por establo fue de 56 vacas, $21 \%$ de los establos estuvieron por encima del promedio y que el $79 \%$ estuvieron por debajo del promedio general. Los resultados tuvieron diferencias altamente significativas $(\alpha=0.01)$, con respecto al número total de vacas entre los establos en evaluación, siendo el máximo promedio por establo de 379 vacas y el promedio mínimo de siete vacas. Es decir extremos muy distantes en forma similar al tamaño de las parcelas.

\section{Cantidad y porcentaje de vacas en producción Por años}

En el Tabla 1 se muestran la cantidad y porcentaje de vacas en producción para el periodo correspondiente a los años del 2005 al 2010, los cuales fueron: 1,117; 1,$322 ; 1,249 ; 1,345 ; 1,256$ y 1,321 vacas, representando los siguientes porcentajes: $86 \%, 65 \%, 88 \%, 85 \%, 84 \%$ y $86 \%$, respectivamente, siendo el total para el período de años en estudio de 7,610 vacas, que representan el $81 \%$ de vacas en producción, el porcentaje de vacas en seca $19 \%$. Estos resultados son similares al encontrado por Iglesias (2006) de 83\% para la Irrigación de Majes en la sección "A". Sin embargo, son inferiores al $88 \%$ reportado por Pallete (2005) para la Cuenca de Lima. Esta diferencia se explicaría en el tipo de explotación más intensiva que se tiene en la cuenca lechera de Lima.

No se encontró diferencia significativa (n.s), entre los años del periodo en estudio, para cantidades totales de vacas en producción y pero si entre los porcentajes que estos representan, el número mínimo de vacas en producción se halló en el año 2005 siendo de 1,117 vacas y el número máximo fue de 1,345 vacas en producción en el 2008;

\section{Por establos}

En el Tabla 2, se muestran los resultados del número de vacas en producción de los 29 establos evaluados, que fue en promedio de 45 vacas, el $79 \%$ de los establos tuvieron promedios menores a este y el $21 \%$ de estos un número mayor a dicho promedio. Estos resultados son similares a los encontrados para el total de vacas.

El promedio general del porcentaje de vacas en producción de $81 \%$, similar a los reportados por Cornejo (2005) en el establo Agroinca PPX de 83\% para vacas Holstein y $81 \%$ para Jersey y por Iglesias (2006) que determinó en la Irrigación de Majes, en la sección " $C$ ", un promedio de $83 \%$ de vacas en producción. Hubo diferencia altamente significativa $(\alpha=0.01)$ con respecto a el número de vacas en producción por establos.

En la Figura 2 se puede apreciar la cantidad de vacas total y en producción por establo, notándose que la gran mayoría de establos (79\%) están por debajo del promedio de 56 y de 45 vacas total y en producción respectivamente.

\section{Por Establos}




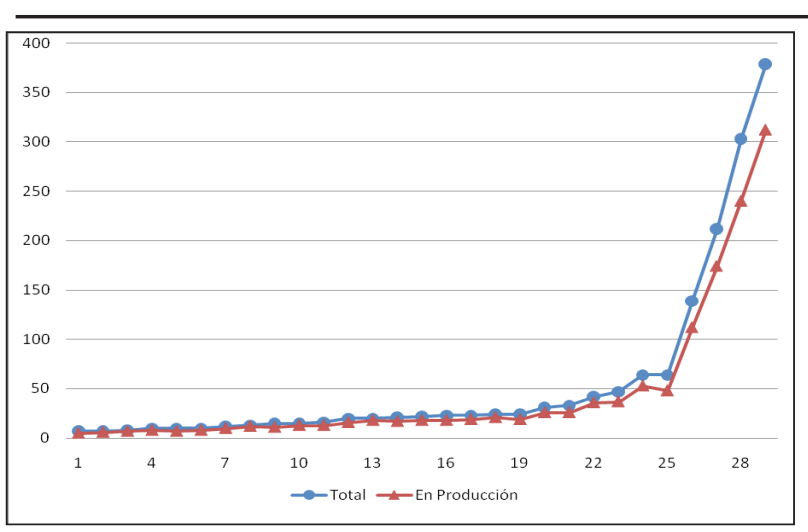

Figura 2. Cantidad de vacas por establo

Tabla 2. Índices productivo por establos

\begin{tabular}{|c|c|c|c|c|c|}
\hline \multirow{2}{*}{ Establos } & \multicolumn{3}{|c|}{ Cantidad de Vacas } & \multicolumn{2}{|c|}{$\begin{array}{l}\text { Producción de } \\
\text { Leche }\end{array}$} \\
\hline & Total & Producción & Porcentaje & $\begin{array}{c}\text { General } \\
\mathrm{Kg}\end{array}$ & $\begin{array}{c}\text { Por vaca } \\
\mathrm{Kg}\end{array}$ \\
\hline 391 & 16 & 13 & 81 & 12,5 & 14.6 \\
\hline 413 & 379 & 312 & 82 & 22,1 & 26,8 \\
\hline 443 & 303 & 240 & 79 & 21,7 & 27,4 \\
\hline 527 & 212 & 174 & 82 & 17,2 & 21,2 \\
\hline 557 & 24 & 21 & 88 & 24,3 & 27,9 \\
\hline 594 & 139 & 112 & 81 & 19,5 & 24,0 \\
\hline 596 & 64 & 53 & 83 & 14,6 & 17,6 \\
\hline 600 & 22 & 18 & 82 & 14,7 & 17,4 \\
\hline 601 & 47 & 37 & 79 & 15,1 & 19,5 \\
\hline 833 & 13 & 12 & 92 & 22,2 & 25,0 \\
\hline 880 & 23 & 18 & 78 & 10,4 & 13,3 \\
\hline 1357 & 10 & 8 & 80 & 12,4 & 15,3 \\
\hline 1360 & 20 & 16 & 80 & 13,7 & 16,9 \\
\hline 1451 & 15 & 11 & 73 & 10,4 & 14,0 \\
\hline 1454 & 24 & 19 & 79 & 12,3 & 15,0 \\
\hline 1496 & 12 & 10 & 83 & 11,6 & 14,7 \\
\hline 1563 & 20 & 18 & 90 & 12,7 & 14,1 \\
\hline 1734 & 10 & 7 & 70 & 11,9 & 16,1 \\
\hline 1735 & 21 & 17 & 81 & 16,5 & 19,6 \\
\hline 1736 & 10 & 8 & 80 & 11,8 & 15,9 \\
\hline 1737 & 31 & 26 & 84 & 13,7 & 16,3 \\
\hline 1740 & 42 & 36 & 86 & 18,3 & 21,1 \\
\hline 1742 & 33 & 56 & 79 & 15,0 & 19,0 \\
\hline 1744 & 15 & 13 & 87 & 12,5 & 14,2 \\
\hline 1746 & 23 & 19 & 83 & 12,8 & 15,1 \\
\hline 1747 & 64 & 48 & 75 & 16,1 & 21,8 \\
\hline 1748 & 7 & 5 & 71 & 13,1 & 17,3 \\
\hline 1749 & 8 & 7 & 88 & 13,8 & 15,7 \\
\hline 1751 & 7 & 6 & 86 & 15.0 & 17.7 \\
\hline
\end{tabular}

\section{Producción de leche: general \\ Por años}

En el Tabla 1 se aprecia los promedios generales (Hato o Establo) de producción de leche para el periodo de los años 2005 al 2010, los resultados hallados fueron de 14,1; 14,5; 19,$3 ; 19,3 ; 20,0$ y $19.6 \mathrm{Kg} /$ día respectivamente, siendo el promedio máximo de $20 \mathrm{Kg}$ /día en el año 2009 y el promedio mínimo reportado de 14,1 Kg/día en el 2005.

El promedio general para el periodo de seis años fue de $17,7 \mathrm{~kg}$ de leche. Esta cantidad es superior al promedio reportado en la Irrigación Santa Rita en el año 1990, de 11,2 $\mathrm{Kg}$ /día citado por Pimentel (1994), y muy similar al 18,4 $\mathrm{Kg}$ /día reportado por Pallete (2005) para la Cuenca de Lia. Esto es una clara indicación de que los ganaderos de Santa Rita han continuado incrementando sus producciones a través de los años.

Hubo diferencia altamente significativas $(\alpha=0.01)$ con respecto al promedio general de leche por años. Lo que muestra que desde el año 2005 al 2010 el promedio general de leche vario significativamente, siendo esta en forma ascendente. Entre el año 2006 al 2007 se observó un incremento importante en el promedio general de producción, de 4,8 $\mathrm{Kg}$ /día y una disminución en el número total de vacas, lo que significa que los ganaderos efectuaron una mayor selección (saca) para incrementar su nivel de producción. En la Figura 3, se pueden apreciar que las producciones de leche general y por vaca, presentan una tendencia en general positiva, a través del periodo de seis años.

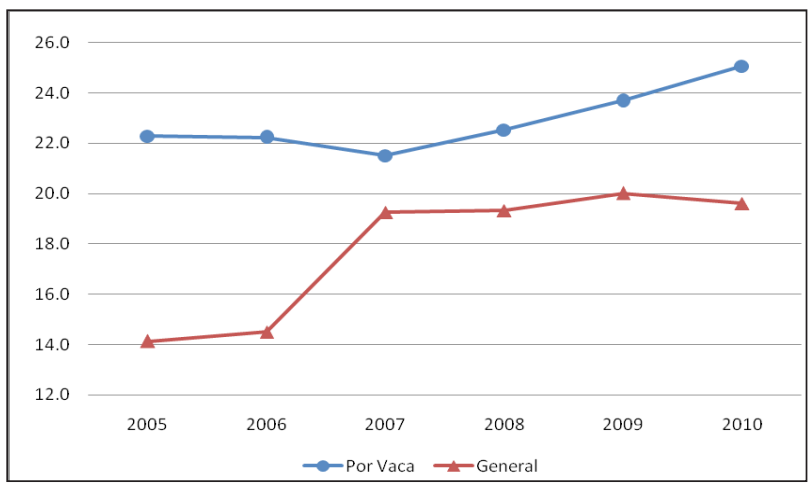

Figura 3. Producción por año

\section{Por establos}

En el Tabla 2 se puede apreciar que los promedios máximos de producción general de leche encontrados fueron de $24,3 \mathrm{Kg}$ /día para un establo con un número total de 24 vacas y de 22,1 Kg/día para uno con 379 vacas y los promedios mínimos para dos establos con promedio general de producción de $10,4 \mathrm{Kg}$ /día con un total de vacas de 23 y 15 respectivamente. Estos resultados nos muestran que los máximos promedios generales de producción de leche fueron encontrados en establos diferentes en cuanto a número total de animales, pero que los promedios más bajos se dieron en establos similares en cuanto a este parámetro, lo que significa que los promedios de producción de leche en los establos de Santa Rita no varían por el número total de animales, observándose que tanto los establos grandes como los pequeños son capaces de alcanzarlos máximos de producción de su zona. Hubo diferencia altamente significativa $(\alpha=0.01)$ en la producción general entre los establos evaluados.

El promedio general de leche para los 29 fue de 18,5 $\mathrm{kg}$. Este promedio general fue menor que el reportado por Cornejo (2005) de 24,63 Kg/día, en el establo Agroinca 
PPX de la Irrigación de San Camilo, pero es similar al promedio máximo por establo de Santa Rita que fue de 24,3 Kg/día.

En la Figura 4 se puede apreciar las producciones de leche general de los establos evaluados y que varían de $10,4 \mathrm{~kg}$ a $24,3 \mathrm{~kg} /$ día.

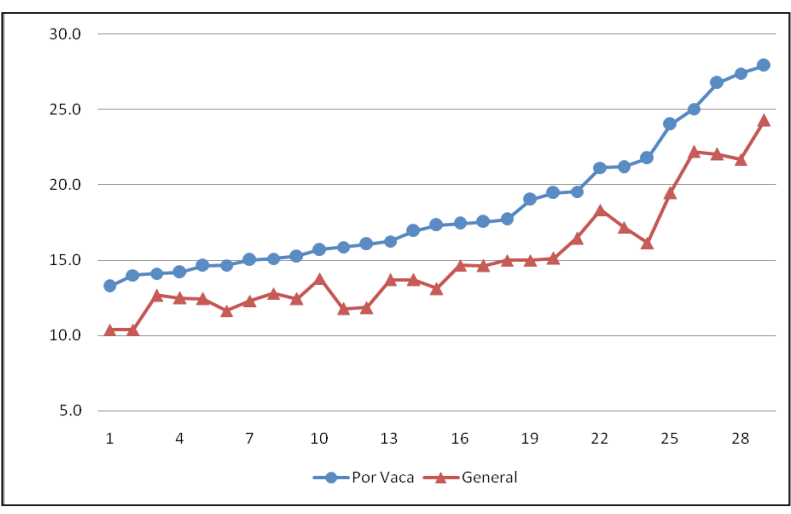

Figura 4. Producción por establo

\section{Producción de leche: por vaca \\ Por años}

En el Tabla 1 se muestran los promedios por vaca en producción para el periodo de años en estudio, encontrándose de promedio mínimo $21,5 \mathrm{Kg}$ /día en el año 2007 y de promedio máximo 25,1 Kg/día en el 2010. Los promedios hallados en el periodo del 2005 al 2010 fueron: 22,$3 ; 22,2 ; 21,5 ; 22,5 ; 23,7$ y $25,1 \mathrm{Kg}$ /día respectivamente, habiendo diferencia altamente significativas $(\alpha=0.01)$ entre los años de evaluación, mostrando una tendencia ascendente a través de los años.

El promedio de leche por vaca para todo el período de seis años fue de $22,9 \mathrm{~kg}$ de leche, este promedio de producción de leche por vaca, es mayor a lo reportado por Bernal (1993) quien encontró un promedio de 14,5 Kg/ día para las Irrigaciones de La Joya, Yuramayo, La Cano, San Camilo y San Isidro y también que los encontrados por Pimentel (1994) que reportó el promedio de 15,7 $\mathrm{Kg}$ /día en la Irrigación de Majes en la Sección “A”. Así mismo se comparan favorablemente a los reportados por Pallete (2005) de $16,7 \mathrm{Kg}$ a $23,4 \mathrm{Kg}$ de leche por vaca en producción en la Cuenca de Lima. Las variaciones de promedios de producción de leche $\mathrm{kg} /$ día a través de los seis años del estudio se pueden apreciar en la Figura 3.

\section{Por Establos}

En el Tabla 2, se muestra los promedios por vaca en producción por establos siendo el máximo encontrado de $27,9 \mathrm{Kg}$ /día y un mínimo de 13,2 Kg/día por establos.

Para los 29 establos el promedio por vaca fue de 22,8 $\mathrm{Kg}$ de leche esta cifra es superior al 20,8 Kg reportada por Pallete (2005) para la Cuenca de Lima. Se encontró diferencia altamente significativa $(\alpha=0.01)$ en el promedio de producción por vaca entre los establos.
Estas variaciones entre establos de 13,3 a 27,9 kg/día por vaca en producción se pueden apreciar en la Figura 4.

\section{Conclusiones}

Los ganaderos de Santa Rita de Siguas en su gran mayoría son propietarios de los terrenos en los que están ubicados sus establos y una buena proporción de sus familias están involucradas en dicha actividad; siendo el tamaño de las propiedades una de sus principales ventajas ya que les permite autoabastecerse de alimentos para el ganado. Sin embargo, se encontró diversidad en los establos en cuanto a cantidad de animales y niveles de producción de leche Los promedios productivos tuvieron un incremento a lo largo de los años en que se hizo la evaluación.

\section{Literatura citada}

Bernal, J. 1993. Caracterización de la Ganadería Lechera del Sur - Arequipa (Irrigaciones el Cural, La Joya, La Cano, Yuramayo, Santa Rita y Majes) (Tesis de Ingeniero Zootecnista). Universidad Nacional Agraria La Molina, Lima Perú.

Casapía, R. 2001. Caracterización de la ganadería lechera en 5 secciones de la Irrigación Majes Arequipa. (Tesis) UCSM Programa de Medicina Veterinaria y Zootecnia.

Cornejo, B. 2005. Evaluación comparativa de los índices técnicos y costos de producción de vacunos de la raza Jersey y Holstein Friesian en una explotación intensiva en la Irrigación San Camilo - Arequipa. (Tesis de Ingeniero Zootecnista). Universidad Nacional Agraria La Molina, Lima Perú.

Iglesia, R. H. 2006. Vida Productiva y Principales Causas de Descarte de Vacas Holstein de la sección C, distrito de Majes. (Tesis Médico Veterinario Zootecnista). Universidad Católica de Santa María, Arequipa, Perú.

Medina, M. 1988. Estudio de la Cuenca Lechera de Arequipa (Tesis de Ingeniero Zootecnista). Universidad Nacional Agrarai La Molina, Lima Perú.

Pallete, P.A.; García, S.M. y Cespedes, E.P. 2005 Características de Productividad de Vacas en la Cuenca Lechera de Lima, Reunión Científica Anual XXVIII de la Asociación Peruana de Producción Animal. APPA Iquitos - Perú.

Pimentel, E. 1994. Caracterización de la ganadería lechera en el proyecto Majes Arequipa (Tesis de Ingeniero Zootecnista). Universidad Nacional Agrarai La Molina, Lima Perú.

Valenza, S. J. 1991. Características de la Ganadería Lechera del Sur - Arequipa. (Tesis de Ingeniero Zootecnista). Universidad Nacional Agraria La Molina, Lima Perú. 